\title{
EFFECT OF FUZZY LOGIC CONTROLLER IMPLEMENTATION ON A DIGITALLY CONTROLLED ROBOT MOVEMENT
}

\author{
Azura Che Soh*, Erny Aznida Alwi, Ribhan Zafira Abdul Rahman, Li Hong Fey \\ Department of Electrical and Electronic, \\ Faculty of Engineering, Universiti Putra Malaysia, \\ 43400 Serdang, Selangor, Malaysia. \\ *Corresponding Author: azura@eng.upm.edu.my \\ Received 14 September; Revised 21 September
}

\begin{abstract}
Advanced intelligent control design cannot entirely replace the application of conventional controller in robotic system. On the other hand, intelligent controllers can be implemented into conventional controller design to increase its performance and ability. Most of intelligent control in movement control involves fuzzy logic and neural network system. This study features the influence of fuzzy logic controller upon the performance of robot movement simulation, which is controlled by a digital controller. Model design and simulation are done in SIMULINK, using Fuzzy Logic Toolbox provided by MATLAB software to control the movement speed of a robotic designed system. Finally, the movement speed is found better off controlled with the additional of fuzzy logic controller instead of the digital controller solely.
\end{abstract}

Keywords: Intelligent Control, Fuzzy Logic, Digital Controller, Robot Movement, Simulink.

\section{INTRODUCTION}

Robotics system design is becoming one of challenging tasks for engineers. Moving toward the technology of artificial intelligent robotic designed system, software simulations and control is very important before engineers can proceed with the mechanical and electronic construction of the whole system [1]. Design and development in robotic system has become an important field in engineering industries due to the requirement of high performance autonomous machine to increase production and handle duty under tough environment

In the development of artificial intelligent human bases robotic design system, fuzzy logic controller is very important when dealing with uncertainty in the working condition. Unknown moving path condition and imprecise input signal are two common problems that occur in the real world robot operations. Because of this, fuzzy logic controller is implemented to the system design in order to handle these conditions. A few robotic fuzzy logic controlled design done by previous researcher with various application can be found in [2],[3],[4],[5],[6],[7].

In this research, the system is specifically designed to define the robotic movement. Generally, the design task can be divided into movement system, sensors system, power system, controller circuits and mechanical construction of the robot. However, only movement, sensor and controller system designs are involved in this simulation. Robotic movement system can 
be designed using DC servo motors, AC motor or pneumatic system. Motors are normally used for joint and robot's wheel construction because of its small size and easier to control.

With the fuzzy logic control blocks [8], the designed system can be improved so that it can handle imprecise signals which are very common in the real world. Whenever the signals alter, the speed of the robotic system is adjusted using the fuzzy logic controller to give an appropriate movement correction defined for the whole system.

Simulation and analysis of this robotic movement control system provide designer with information of how the fuzzy logic controller system can affect the output speed and stability of the system. From this information, designer can proceed with a larger design with more complex controller system.

\section{OBJECTIVES}

This research focused on designing a fuzzy logic controller that is suitable for controlling the movement of robotic system. A fuzzy logic controller is developed and attached into the design, to control the speed of the robot under specified direction and during dynamic changes. A SIMULINK block diagram and an interactive graphical user interface (GUI) system have been developed to simulate and analyze the performance and stability of the robotic movement control system. This study features the influence of fuzzy logic controller upon the performance of robot movement simulation, which is controlled by a digital controller.

\section{METERIALS AND METHOD}

The robotic movement control system designed for this project is based on few subsystems normally available in most digitally controlled movement design technology. The speed system is the DC motor subsystem. This system can be divided into two parts, the mechanical system and electrical system. Both systems are designed to be controlled by a digital controller subsystem.

The sensor subsystem is designed to interact with the digital controller subsystem. The sensor subsystem provides the digital controller subsystem with all information or signals about the environment condition. From this information or signals, the digital controller is expected to be able to control the DC motor subsystem rotation speed. Input switching subsystem is designed to turn on or turn off the motor.

Implementation of fuzzy logic based controller system is another approach to this design. This controller will interact with the sensor signal and provide additional control to the DC motor subsystem. The designed robotic movement control system block diagram is shown in Fig.1.

The DC motor subsystem is the main component that decides how fast the robotic system will move. This subsystem is connected to the mechanical movement system of the designed robot. The main idea in this simulation is to observe how the speed of the robotic system can be controlled and how does a fuzzified controller affect the system. Speed control of a robotic design is always based on how or which type of motor system to be developed. Various types of motor can be used to move the robot joint, robot base system, robot arm and etc. For a simple design, designers normally choose to use either DC servomotor or step motor. In this 
study, digital controlled DC motor model was chosen for the robotic speed control system design.

Basic structure of a DC motor can be divided into two part; voltage controlled circuitry and mechanical rotor. The motor torque provides the movement over inertia for the rotor system. If the motor initially in static condition, it requires larger torque value to start the motor. If the motor initially in moving condition, the inertia of the motor will sum up with the system torque to give a grater rotation which will further increase the moment speed of this system.

The velocity of the motor can be defined as the rate of change in rotor position, $\theta$.

$$
\text { Motor Velocity }=\frac{d \theta}{d t}
$$

From the study of Newton's law, the motor rotation angle characteristic can be defined as:

$$
J \frac{d^{2} \theta}{d t^{2}}=T-b \frac{d \theta}{d t}
$$

Whereby:

$J=$ moment of inertia of the rotor, $T=$ motor torque, $B=$ damping ratio of the mechanical system, $\frac{d^{2} \theta}{d t}=$ rotational acceleration,$\frac{d \theta}{d t}=$ rotational velocity.

This equation for motor rotation characteristic was further simplified to:

$$
\frac{d^{2} \theta}{d t^{2}}=\frac{1}{J}\left(K_{t} i-b \frac{d \theta}{d t}\right)
$$

The second equation need to be taken into consideration is the DC motor equivalent circuit characteristic equation. Using the Kirchoff's Law, the circuit equivalent equation can be developed as shown below:

$$
\begin{array}{ll}
\text { Start: } & L \frac{d i}{d t}=-R i+V-e \\
& \frac{d i}{d t}=\frac{1}{L}\left(-R i+V-K_{e} \frac{d \theta}{d t}\right)
\end{array}
$$

Whereby:

$L$ is electric inductance, $R$ is electric resistance, $V$ is applied voltage source and $K_{e}$ motor constant.

The DC motor subsystem model is designed using SIMULINK block diagram as shown in the Fig.2. From the figure, a few blocks are set as constant parameters namely the $\mathrm{K}$ (electromotive force constant), $\mathrm{R}$ (electric resistance), $\mathrm{J}$ (moment of inertia of the rotor), $\mathrm{L}$ (electric inductance) and $\mathrm{b}$ (damping ratio of the mechanical system). The parameters' value 
used for this simulation is taken from an experiment done on an actual motor in Carnegie Mellon's undergraduate controls lab [9].

Moment of inertia of the rotor $(\mathrm{J})=0.01 \mathrm{kgm}^{2} \mathrm{~s}^{-2}$

Damping ratio of the mechanical system $(\mathrm{b})=0.1 \mathrm{Nms}$

Electromotive force constant $\left(\mathrm{K}=\mathrm{K}_{\mathrm{e}}=\mathrm{K}_{\mathrm{t}}\right)=0.01 N m A m p^{-1}$

Electric resistance $(\mathrm{R})=1 \Omega$

Electric inductance $(\mathrm{L})=0.5 \mathrm{H}$

Designing the digital controller for this system require additional Zero Order Hold blocks added to both of the input and output block. The first Zero Order Hold block connected to the input block is used to convert a discrete-time signal to a stepwise-constant continuous signal and the second Zero Order Hold connected to the output block is used to take discrete samples of the output from the subsystem (Fig.3). The Sample Time Field of each block was set to $0.001 \mathrm{~s}$ which have faster response than the MATLAB desired step response signal. This will give a approximate continuous signals as required in the design.

Details about the digital controller system are not taken into consideration in this study. From previous study by Carnegie Mellon's undergraduate controls lab [9], the digital controller of this system can be represented in a transfer function as shown below:

$$
\text { Digital Controller }=450 \frac{(z-0.85)(z-.085)}{(z+0.98)(z-0.7)}
$$

Feedback system with unity gain is designed for the feedback loop of the digital controlled motor system as shown in Fig.4. This model can be used to represent the basic movement system of a robotic design. Any robotic system using DC source controlled motor require similar system as shown in this design. The only thing that maybe different is the parameter value of each part or block of the model.

Fuzzy logic controller block was implemented to this system to give fuzziness control over the digitized control signal. Two types of fuzzy controller model were introduced for intelligent design, namely the Mamdani fuzzy controller model [10] and the Sugeno fuzzy controller model [11]. For robotic movement simulation design, various possible output and input data have to be taken into consideration. In this case the Sugeno fuzzy model is not suitable because of the output membership function limitation. Due to this reason, the Mamdani fuzzy controller model which provides a wide spread membership function for the output was chosen for this design.

The first input of the FIS controller is the sensor signals. In a normal electronic design, the sensors signals are ranged from $0 \mathrm{~V}$ to $5 \mathrm{~V}$. Because of this, the weight of the input is set initially ranged from 0 to 5 . Signals from the sensor are subdivided into five ranges; very weak signal, weak signal, medium signal, strong signal and very strong signal. The second input of the FIS controller is the rate of change in the sensor's signal. In this case, the signals are assumed to change positively and negatively. Initially the signals change rate is zero. When the sensor's signals increases proportionally to the time period (sensor's signal become 
stronger), the sensor's signal rate is said to change positively. If the sensor's signal decreases proportionally to the time period (sensor's signal become weaker), the sensor's signal rate is said to change negatively.

The main consideration of this robotic design is the movement speed of the robot. In this case, the designed system considers that the robot is moving toward the target. The output is designed to have weight of 10 and uses the triangular-shaped built-in membership function. Weight of the output represents the speed of the robot, respectively the speed of the motors in the robotic design. Designed output is further subdivided into six ranges; stop range, very slow speed, slow speed, medium speed, fast speed and very fast speed.

The summation of the fuzzy controller block and the digital controller block provide the total input of the motor subsystem. The SIMULINK block diagram for the whole robotic system with fuzzy controller is shown in the diagram below in Fig.5.

To design the fuzzy logic controller rules based system, all input conditions need to be taken into consideration. In the rule editor, all the possible fuzzified input and output criteria are shown in the rules development boxes. Using the "IF-AND/OR-THEN" [7] condition, all the required rules for fuzzy controlled system can be established. Base on the condition defined in the Table 1 above, the rules of the initially designed robotic system is generated.

When the setting is done, the SIMULINK block diagram is ready to be simulated to get the required output result. From the SIMULINK result, designer can observe and analyze the fuzzy controller output signals, sensor signal and the movement speed of the whole system. This result is compared to the system designed without fuzzy controller.

\section{RESULT AND DISCUSSIONS}

The designed system will be simulated under three different environmental conditions, i.e. increasing, decreasing and constant sensor signal voltage rate which represent robot movement approaching obstacle, moving away from obstacle and has a constant range from the obstacle respectively. For simulation, the input signal of the robotic system is fixed to $5 \mathrm{~V}$ (consider for normal digital control integrated circuit logic ' 1 ' input). The sampling time of the system is assumed $0.01 \mathrm{~s}$ and the signal rate is maintained at $0 \mathrm{Vs}^{-1}$ for $0.01 \mathrm{~s}$ or the step time is set to $0.01 \mathrm{~s}$. The simulation time is set to $1.0 \mathrm{~s}$. Simulation results will be compared and discussed.

\section{Robotic system approaching obstacle}

In this case, the robotic system is assumed to move toward the obstacle. The signal rate is increasing from $0 \mathrm{Vs}^{-1}$ to $1 \mathrm{Vs}^{-1}$. Therefore, the initial voltage for the sensor rate input is set to 0 and the final voltage value is set to 1.The simulation time is set to 1.0 s for this case to view the signal output graph at this specified timing range. While the output below show how velocity of the system changes with time for designed system with fuzzy logic controller system.

From this result shown in Fig. 6, the motor start up at time $t=0$ s for 0.35 second to maximum speed of 1.75 units/s. The robotic movement system speed will decrease rapidly when the sensor signal voltage rate is step up to 1 . The robotic movement decelerates when it moves 
toward the obstacle. The velocity of the robot will reach zero when in $0.74 \mathrm{~s}$ time from the starting time. At this point the system stops and turns to move in the opposite direction in the increasing velocity (speed increased in negative unit).

Compared to the system without fuzzy logic controller in Fig.7, this system will reach higher velocity before it decelerates. From this result, the system with fuzzy logic controller is seemed to have higher startup rate. This robotic system will stop and change the movement direction when reach 0.76 second, 0.02 second slower than previous model. Designer should take this statement into design consideration to estimate the robot-obstacle range before the robotic system crash into the obstacle. When the sensor signal rate is increasing with time, the sensor output signal is also increased linearly.

\section{Robotic system moving away from obstacle}

For this simulation analysis, the input signal of the system is maintained at step voltage of $5 \mathrm{~V}$ as what have done in the previous system. The sensor signal rate is now decreasing to negative value. The sensor signal rate is assumed to change from $0 \mathrm{Vs}^{-1}$ to $-1 \mathrm{Vs}^{-1}$ in a step condition with the step time of 0.01 second. Thus, the initial value of this input is set to 0 and the final value is set to -1 .

The robotic movement system without fuzzy logic controller output is shown in Fig. 8 . Whereas, the output characteristic for robotic movement system with fuzzy logic controller for decreasing sensor signal rate condition is shown in Fig.9. The system velocity increases rapidly in this case. The speed can reach up to 8 units in $1 \mathrm{sec}$ time. The motor is having acceleration in this case. From this result, the velocity of the system is increasing linearly inverse proportional to the sensor input voltage. The fuzzy logic controller system improves the output velocity performance by increasing the output voltage magnitude at each sample time by 0.025 units/s larger than the normal system. The sensor output voltage decreases when the sensor signal rate is decreasing. This will increase the total input voltage of the designed system and further increase the robotic movement system speed.

The fuzzy logic controller output seems to decrease when the output velocity increases in high speed. This shows that the fuzzy logic controller is trying to pull down the system velocity when it goes higher. This control signal can provide auto protection to the system whereby the output velocity was limited to a certain value by the fuzzy logic controller system.

\section{Robotic system has a constant range from obstacle}

Assume the system has lost the obstacle under certain condition. Because of this the sensor signal rate is decreasing from $1 \mathrm{Vs}^{-1}$ to $0 \mathrm{Vs}^{-1}$ at a set step time of 0.01 second. The sensor signal rate output parameter is modified to give a constant sensor signal input voltage to the system voltage. To do this, the initial value of the sensor signal input rate is set to 1 and final value is set to 0 . The output voltage characteristic and sensor signal voltage output for the robotic movement system is shown in the scope output of Fig. 10 and Fig. 11.

The robot velocity increases from 0 units/s to 3.1 units $/ \mathrm{s}$ in $0.65 \mathrm{~s}$. The robot is having acceleration in this case. After $0.6 \mathrm{~s}$, the velocity of the robot is maintained at $3.1 \mathrm{units} / \mathrm{s}$. The robotic system is assumed to be stable at this condition because the output velocity is not 
decreasing or increasing rapidly. The velocity output for system with fuzzy logic controller is 0.03 units higher. This system works on under damped condition because it does not reach the maximum input value. The result shows that the system provides higher velocity with same input voltage, which will increase the overall system performance. From here, the robotic movement system with fuzzy logic controller can provide shorter startup time to the system.

\section{Step response analysis}

For this analysis, DC motor and controller parameters are set to the default value. The step response for the robotic movement system with fuzzy logic controller and without fuzzy logic controller is shown in the Fig. 12 and Fig. 13.

Obviously, the robotic movement system without fuzzy logic controller shows a negative response. In this case, the performance of this system maybe degraded with time. When the fuzzy logic controller is connected to the system, the performance and stability of the system can be much improved over time. This can be seen from the over damping step response output with step of one at stability.

\section{Root locus analysis}

Root-locus analysis is used to describe the change in transient response and to determine the stability of a system. The system is considered to be stable if the root-locus sketch for the specific system does not cross the jw-axis. A stable system will have more poles than zeros.

More point available in the negative side of the root-locus sketch shows that there are more poles and zeros in the system. Fig. 14 and Fig. 15 show the root locus analysis results of both robotic movement systems with fuzzy logic controller and without fuzzy logic controller.

From this root locus analysis result, the critical point of the system without fuzzy logic controller is situated along zero value of the root-locus sketch. Some values can go beyond the positive side of the $\sigma$-axis. This shows that the system is not stable and may have some positive feedback condition. From the root-locus sketch output for the robotic movement system with fuzzy logic control, all critical points are located at the negative side of the $\sigma$-axis. This shows that the system have a negative feedback control and stable electrically.

\section{Open loop analysis}

Open loop analysis is used to check the system response when there is no feedback. Fig. 16 and Fig. 17 show the open loop analysis results of both robotic movement systems with fuzzy logic controller and without fuzzy logic controller. The robotic system without fuzzy logic controller shows negative damping gain of 10 during the open loop analysis. This shows that the system is critically unstable. To increase the system stability, designer should implement a large gain negative feedback system into this design.

Another alternative from using large negative feedback system is to use fuzzy controller. From the open loop analysis result, the open-loop damping gain is zero for the robotic movement system designed with fuzzy logic controller. This shows that the system is highly stable after a period of time. 


\section{CONCLUSIONS}

Implementation of fuzzy logic controller in robotic movement control system can improve overall output speed performance. Two different robotic movement control systems, i.e. with and without fuzzy logic controller have been analyzed and the output characteristics were compared. From the analysis, the system with fuzzy logic controller is found to be more efficient, where it improves the speed and increases the stability of the system. Yet, the performance of the robotic movement control system with fuzzy logic controller can be further improved by adding the neural network fault detection system. With this, the intelligence of the designed system can be increased.

\section{ACKNOWLEDGEMENT}

The work reported here is part of short term research work funded by the Universiti Putra Malaysia.

\section{REFERENCES}

1. Azura Che Soh, Mohd Khair Hassan and Li Hong Fey 2004. "Intelligent movement control for robots using fuzzy logic", Conference Artificial Intelligence in Engineering and Technology(ICAET-2004), Sabah, Malaysia.

2. Nazim, N. M. and Salami M.J.E 1999. "Design of intelligent multifinger robotic gripper using slip detection technique", Proc. World Engineering Congress (WEC'99), pp. 17-21,

3. Chor, K. S., Hamouda A. M. S., Wong, S. V. and Napsiah Ismail, "An intelligent mobile robot for fire detection in virtual world", Study by Institute of Advance Technology (ITMA) and Department of Mechanical and Manufacturing Engineering, University Putra Malaysia, 2003.

4 Rajasekharan S. and Kambjampati, C., "Control of cooperating robots: conventional or intelligent", Proceedings of the 7th International Symposium on Intelligent Robotic Systems, 1999

5 Castro, N. Matias, R. and Ribeiro, M. I. ,'Target tracking using fuzzy control", Proceedings of the Scientific Meeting of 3rd. Robotics National Festival,2003

6 Habib, M.K. ,'Designing fuzzy logic controllers for DC servomotors supported by fuzzy logic control development environment", Industrial Electronics Society (IECON '01), The 27th Annual Conference of the IEEE. 3: 2093-2098, 2001.

7 Novakovic, B.M., Crnekovic, M. and Oluic, C.,"An analytic fuzzy logic control of robots", Emerging Technologies and Factory Automation Proceedings (ETFA '97), 6th International Conference. pp 403-408,1997 
8 MATLAB Fuzzy Logic Toolbox User's Guide,2001

9 Intelligent System Laboratory, School of Engineering University of Guelph. http://wolfman.eos.uoguelph.ca/ jzelek/matlab/ctms/simulink/examples/motor/motorsi m.htm., 2001

10 Mamdani, E.H. and S. Assilian, "An experiment in linguistic synthesis with a fuzzy logic controller", International Journal of Man-Machine Studies. 7: pp 1-13, 1975

11 Sugeno, M., Industrial applications of fuzzy control, Elsevier Science Pub. Co., 1985.

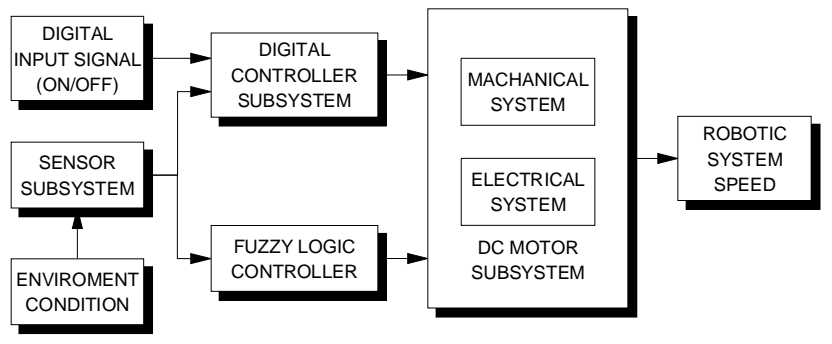

Fig. 1: Block diagram of the robotic movement control system

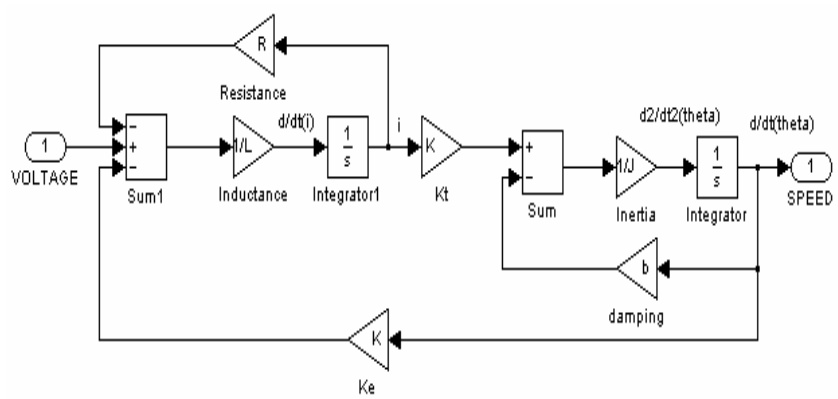

Fig.2: SIMULINK block diagram of the DC motor subsystem

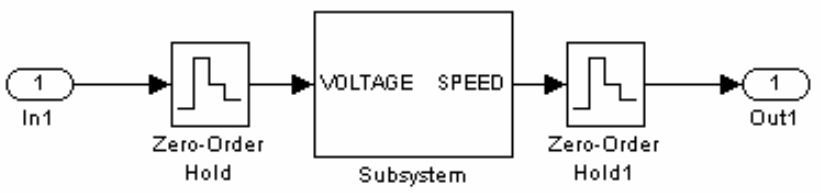

Fig.3: Additional of Zero Order Hold block to the DC motor subsystem for digital control

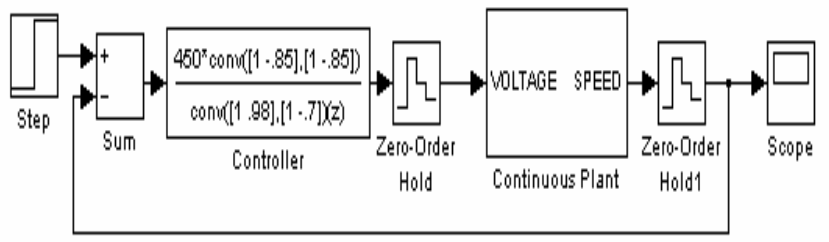

Fig. 4: Robotic movement system with digital controller 


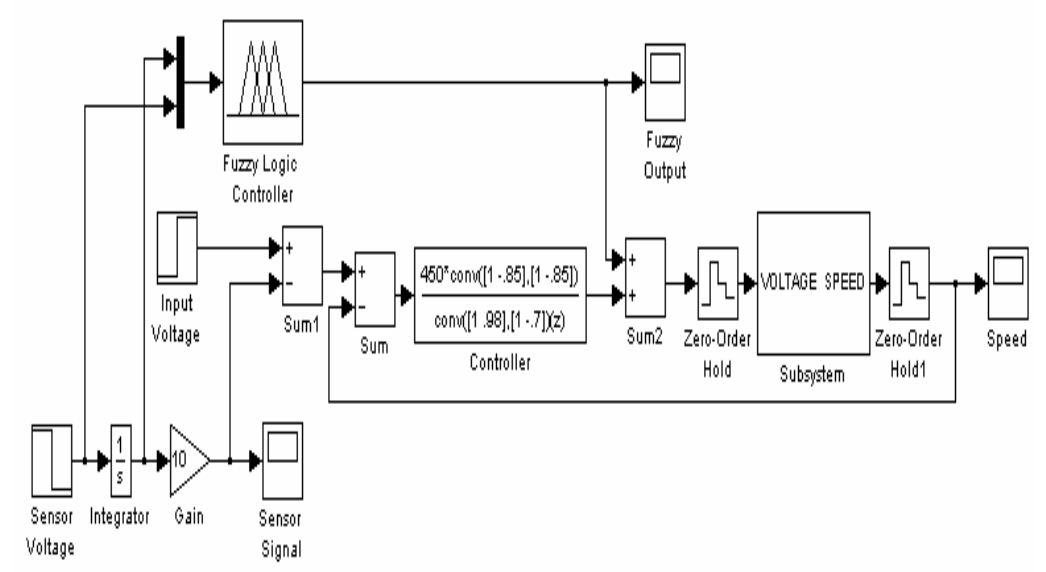

Fig.5: Robotic movement system with fuzzy logic controller block
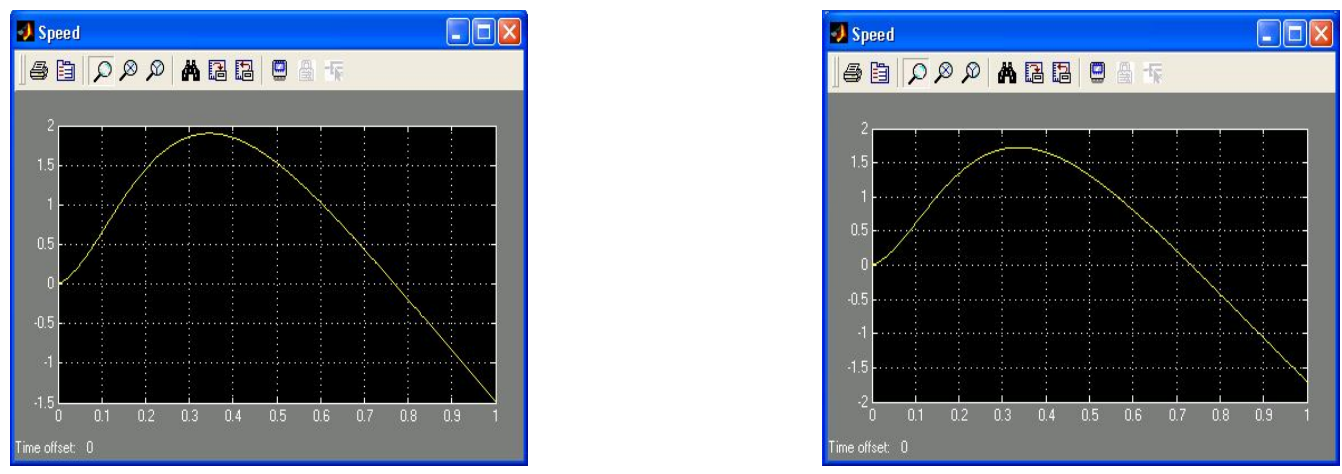

Fig. 6: Robotic model velocity output vs time Fig. 7: Robotic model velocity output vs time (with fuzzy logic controller) (without fuzzy logic controller)

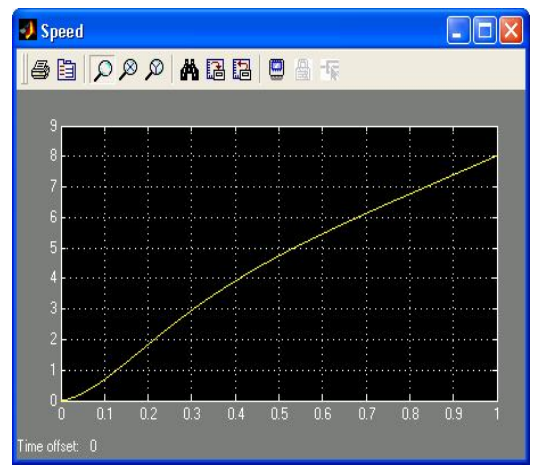

Fig. 8 : Robotic model velocity output vs time (without fuzzy logic controller)

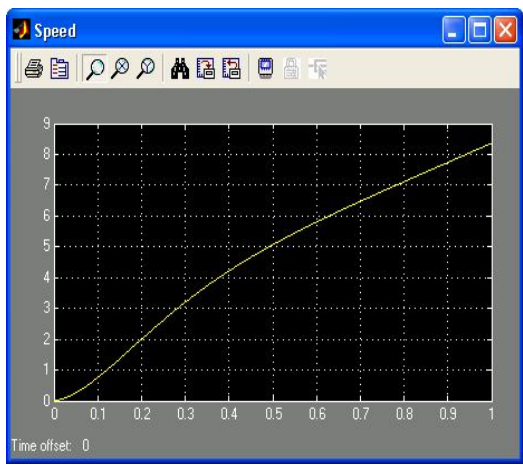

Fig. 9: Robotic model velocity output vs time (with fuzzy logic controller) 


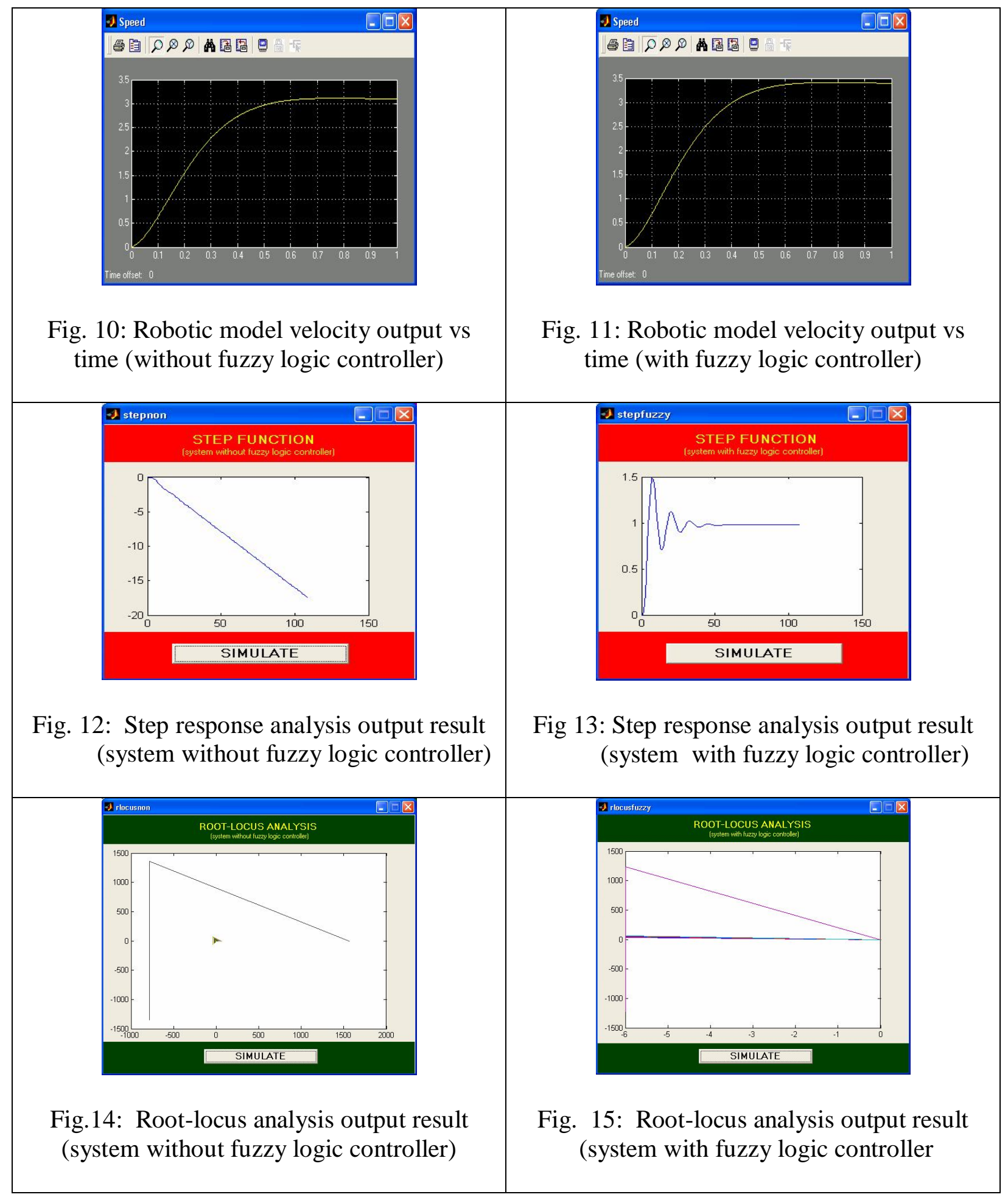




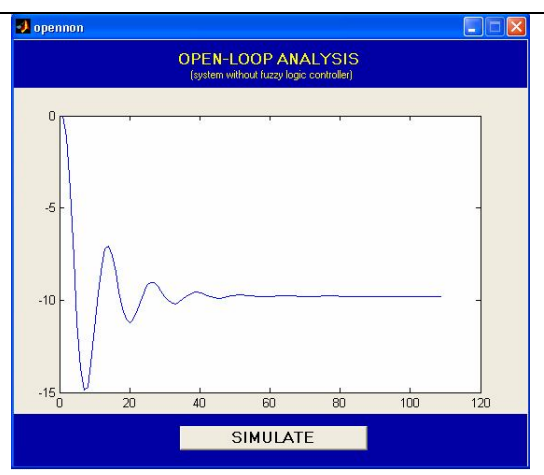

Fig. 16: Open-loop analysis output result (system without fuzzy logic controller)

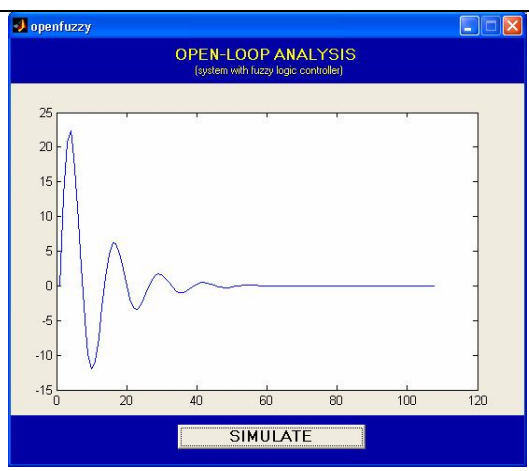

Fig. 17 : Open-loop analysis output result (system with fuzzy logic controller)

Table 1. Fuzzy Logic Controller Designed Rules

\begin{tabular}{cccccc}
\hline \hline IF & $\begin{array}{c}\text { Input 1 } \\
\text { (Signals) }\end{array}$ & Condition & $\begin{array}{c}\text { Input 2 } \\
\text { (Rate of Change) }\end{array}$ & Condition & $\begin{array}{c}\text { Output } \\
\text { (Speed) }\end{array}$ \\
\hline RULE 1 & Very Weak & AND & Negative & THEN & Very Fast \\
RULE 2 & Very Weak & AND & Zero & THEN & Fast \\
RULE 3 & Very Weak & AND & Positive & THEN & Medium \\
RULE 4 & Weak & AND & Negative & THEN & Very Fast \\
RULE 5 & Weak & AND & Zero & THEN & Fast \\
RULE 6 & Weak & AND & Positive & THEN & Medium \\
RULE 7 & Medium & AND & Negative & THEN & Fast \\
RULE 8 & Medium & AND & Zero & THEN & Medium \\
RULE 9 & Medium & AND & Positive & THEN & Slow \\
RULE 10 & Strong & AND & Negative & THEN & Medium \\
RULE 11 & Strong & AND & Zero & THEN & Slow \\
RULE 12 & Strong & AND & Positive & THEN & Very Slow \\
RULE 13 & Very Strong & AND & Negative & THEN & Slow \\
RULE 14 & Very Strong & AND & Zero & THEN & Very Slow \\
RULE 15 & Very Strong & AND & Positive & THEN & Stop \\
\hline \hline
\end{tabular}

